\title{
Two-photon exchange contribution in elastic electron-proton scattering, experiment at the VEPP-3 storage ring
}

D.M. Nikolenko ${ }^{1, a}$, J. Arrington ${ }^{2}$, L.M. Barkov ${ }^{1, b}$, V.F. Dmitriev ${ }^{1,3}$, V.V. Gauzshtein ${ }^{4}$, R.A. Golovin ${ }^{1}$, A.V. Gramolin ${ }^{1}$, R.J. Holt ${ }^{2}$, V.V. Kaminsky ${ }^{1}$, B.A. Lazarenko ${ }^{1}$, S.I. Mishnev ${ }^{1}$, N.Yu. Muchnoi ${ }^{1,3}$, V.V. Neufeld $^{1}$, I.A. Rachek ${ }^{1}$, R.Sh. Sadykov ${ }^{1}$, Yu.V. Shestakov ${ }^{1,3}$, V.N. Stibunov ${ }^{4}$, D.K.Toporkov ${ }^{1,3}$, H. de Vries ${ }^{5}$, S.A. Zevakov ${ }^{1}$, and V.N. Zhilich ${ }^{1}$

\footnotetext{
${ }^{1}$ Budker Institute of Nuclear Physics of SB RAS, 630090 Novosibirsk, Russia

${ }^{2}$ Argonne National Laboratory, Argonne, Illinois 60439, USA

${ }^{3}$ Novosibirsk State University, 630090 Novosibirsk, Russia

${ }^{4}$ Nuclear Physics Institute of Tomsk Polytechnic University, 634050 Tomsk, Russia

${ }^{5}$ NIKHEF, P.O. Box 41882, 1009 DB Amsterdam, The Netherlands
}

\begin{abstract}
We describe a precise measurement of the ratio of the $\left(e^{+} p\right)$ to $\left(e^{-} p\right)$ elastic scattering cross sections. This comparison is sensitive to the effect of two-photon exchange contributions which may be the cause for inconsistent extractions of the proton form factors obtained using different methods. The experiment was performed at storage ring VEPP-3, Novosibirsk at energies of positron/electron beams of 1.0 and $1.6 \mathrm{GeV}$ with electron/positron scattering angles $\theta=65 \div 105^{\circ}$ for the first case and $15 \div 25^{\circ}$ and $55 \div 75^{\circ}$ for the second case. Details of the experiment and the preliminary results are presented.
\end{abstract}

\section{Introduction}

Detailed studies of the electromagnetic form factors of the proton are crucial to the understanding of its structure. These studies help to establish details of interaction of it's constituents - the quarks and gluons. In addition, reliable information about the form factors has an important role in many fields of physics.

The experimental study of proton form factors is performed with elastic electron-proton scattering. Since the discovery of the finite size of the proton, the Longitudinal-Transverse separation or Rosenbluth separation (RS) was used for disentangling of the electric $\left(G_{E}\right)$ and magnetic $\left(G_{M}\right)$ form factors of the proton during the the past several decades. RS is based on the Rosenbluth one-photon exchange formula for the differential cross section [1]:

$$
\frac{d \sigma}{d \Omega}=\left(\frac{d \sigma}{d \Omega}\right)_{M o t t} \times \frac{\tau}{\epsilon(1+\tau)} \times\left[\frac{\epsilon}{\tau} G_{E}^{2}+G_{M}^{2}\right]
$$

\footnotetext{
a e-mail: D.M.Nikolenko@inp.nsk.su

${ }^{\mathrm{b}}$ Deceased.
} 
Table 1. Kinematic settings of two Runs of the Novosibirsk TPE experiment.

\begin{tabular}{|c|c|c|c|c|c|}
\hline \multirow{2}{*}{ Parameter } & \multicolumn{3}{|c|}{ Run I } & \multicolumn{2}{c|}{ Run II } \\
\cline { 2 - 6 } & LA & MA & SA & LA & MA \\
\hline \hline$E_{\text {beam }, \mathrm{GeV}}$ & \multicolumn{3}{|c|}{1.6} & \multicolumn{2}{|c|}{1.0} \\
\hline$\theta$, degree & $55-75$ & $15-25$ & $8-15$ & $65-105$ & $15-25$ \\
\hline$Q^{2}, \mathrm{GeV}^{2}$ & $1.26-1.68$ & $0.16-0.41$ & $0.05-0.16$ & $0.71-1.08$ & $0.07-0.17$ \\
\hline$\varepsilon$ & $0.58-0.37$ & $0.97-0.90$ & $0.99-0.97$ & $0.51-0.18$ & $0.97-0.91$ \\
\hline
\end{tabular}

where $\tau=Q^{2} / 4 M^{2}$ and $\epsilon=\left[1+2(1+\tau) \tan ^{2}(\theta / 2)\right]^{-1}$ virtual photon polarization. In this method one measures the unpolarized (ep)-elastic cross section at constant $Q^{2}$, varying beam energy and $\theta$ electron scattering angle.

In 1968 Akhieser and Rekalo [2] showed that in elastic scattering of polarized electron on proton the ratio of form factors can be related to the ratio of components of the recoil polarization:

$$
\frac{G_{E}}{G_{M}}=\frac{P_{T}}{P_{L}} \times K,
$$

where $P_{T}$ and $P_{L}$ are transverse and longitudinal polarization components of the proton, and $K=-\sqrt{\tau(1+\epsilon) / 2 \epsilon}-$ is a kinematic factor. However, the experiments, using a polarization transfer method, were performed much later, when the high-quality polarized electrons beams and the recoil proton polarimeters became available. Unexpectedly, a clear discrepancy between results of two methods was observed [3]. This discrepancy is a serious problem as it generates confusion and doubt about the whole methodology of lepton scattering experiments.

It is often argued that the origin of this discrepancy is the failure of the one-photon approximation to precisely describe the results of RS experiments. However, consideration of the two-photon exchange (TPE) contribution encounters difficulties of both a theoretical and experimental character [4]. The problem has stimulated many new theoretical studies performed with various approaches as well as the suggestions of new experiments for the determination of TPE contribution to this reaction. The most direct way to determine TPE contribution is a measurement of the positron-proton and electronproton scattering cross sections ratio $R=\sigma\left(e^{+} p\right) / \sigma\left(e^{-} p\right)$. Previous measurements of $R$ have a large uncertainty [5] and new precise data are required to verify the models.

\section{Experiment Descripton}

Here we report the experiment on a precise measurement of $R$, which was performed at the storage ring VEPP-3, Novosibirsk [6]. We performed two runs: Run-I at a $1.6 \mathrm{GeV}$ and Run-II at a $1 \mathrm{GeV}$ beam energy. Kinematic settings are shown in the Table 1. Run-I has three angular ranges and Run-II has two angular ranges. but the smallest angular range was used for luminosity monitoring only. That is because it is generally believed that for small $Q^{2}$ and for $\epsilon \approx 1$ the TPE is very small, i.e. $R \approx 1$

The internal gas target is based on an open-ended storage cell, having an elliptical cross-section $13 \times 24 \mathrm{~mm}^{2}$ and a length of $400 \mathrm{~mm}$. Hydrogen gas is injected into the cell center and provides a target thickness of $\approx 10^{15} \mathrm{~atm} . / \mathrm{cm}^{2}$. The detector is comprised of two nearly identical parts placed symmetrically with respect to the storage ring median plane. The azimuthal angle acceptance of each system is $60^{\circ}$, so the total acceptance is $\Delta \phi=120^{\circ}$. Such a detector configuration helps to suppress systematic uncertainty. 
Scattered positron/electron and recoil proton are detected in coincidence, which facilitates the separation of the elastic scattering events from the various background events by using kinematic correlations. The detector package contained the wire chambers for tracking, plastic scintillators for triggering, for measurement energy of slow protons and multilayer segmented electro-magnetic calorimeter for measurement of the scattered electron energy. The detector is a wide-aperture nonmagnetic apparatus. Carefully chosen material has been placed in front of wire chambers to suppress low energy background.

To select elastic (ep)-scattering events we applied cuts on correlations between different reconstructed values: i) correlation between polar angles of $e^{+}$or $e^{-}$and $p$; ii) correlation between azimuthal angles of $e^{+}$or $e^{-}$and $p$; iii) correlation between $e^{+}$or $e^{-}$scattering angle and proton energy; iv) correlation between positron or electron scattering angle and its energy; v) $\Delta E-E$ analysis for proton identification; vi) time-of-flight analysis for the identification of proton with low energy.

For suppressing the systematic errors it was important to keep identical conditions during data taking with positron and electron beams. Special attention was given to equality of energies, currents and positions of the beams, equality of target thickness, accelerator related background, and stability of the detector efficiency. Positron/electron beams energies were measured regularly and with good accuracy during data taking by a Compton backscattering setup. We had three sources of information on the $e^{+} / e^{-}$beams positions: VEPP-3 beam position monitors, moveable beam scrapers and vertex position reconstruction using the coordinate system of the detector. Combination of the information from these three sources makes it possible to determine the position of beams with an accuracy of 0.1 $\mathrm{mm}$. The total experimental systematic uncertainty should not exceed $0.3 \%$.

A precise account for standard radiative corrections is a crucial task for the analysis. For a wideacceptance non-magnetic detector, the standard prescriptions of soft-photon peaking approximation (SPA) could not be applied. A detailed Monte-Carlo simulation with usage of the event generator ESEPP [7] was performed. In ESEPP more precise formulas than the SPA [8] have been applied.

\section{Preliminary results and Conclusion}

The preliminary results of the Novosibirsk TPE experiment as well as previous data are shown in Figure 1. Only statistical errors are shown. The radiative corrections are taken into account. Some small corrections are not yet applied, so these results are still preliminary. Three theoretical predictions are shown: calculation of Coulomb correction by Arrington and Sick [9], the hadronic TPE calculation by Blunden, Melnitchouk and Tjon [10] and prediction of a model that exploits the analytic properties of the Compton scattering amplitude by Tomasi-Gustafsson et al [12]. It appears that the calculation in Ref. [10] is in best agreement with the data, however it tends toward overestimating the data. Phenomenological extractions of TPE contributions in this $Q^{2}$ range [11] tend to predict somewhat larger effects than the hadronic calculation, but such extractions are not as much reliable at these $Q^{2}$ values, where the discrepancy between Rosenbluth and polarization results is not clear.

In the conclusion:

- The first precision measurement of the ratio $R=\sigma\left(e^{+} p\right) / \sigma\left(e^{-} p\right)$ has been performed. Data taking has been completed, analysis is in progress.

- Systematic errors in VEPP-3 experiment are expected to be small.

- It is very important to carefully include the radiative corrections.

- Preliminary results are presented. They are more close to the calculation [10], which, at higher $Q^{2}$ values, largely resolves the inconsistency of two approaches to the measurement of proton form factors [13]. 

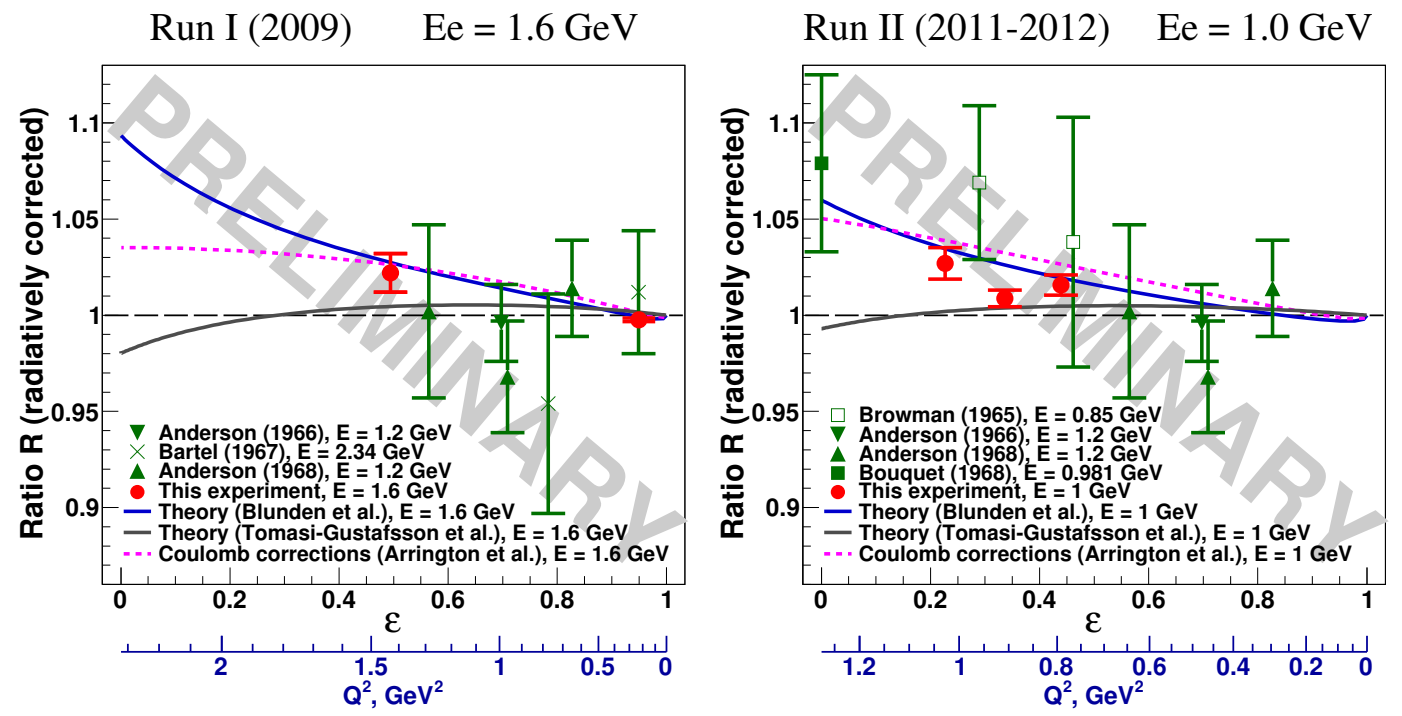

Figure 1. Preliminary results of the VEPP-3 TPE experiment (full circles). Old data obtained with beams of similar energies are also shown. Theoretical curves are described in the text.

This work was supported by Ministry of Education and Science of the Russian Federation; by RFBR, grants: 08-02-00624-a, 08-02-01155-a; by Russian Federal Agency for Education, State Contract P522; by Russian Federal Agency for Science and Innovation, Contract 02.740.11.0245.1; by US DOE, grant DE-AC02-06CH11357; by US NSF, grant PHY-03-54871.

\section{References}

[1] M.N. Rosenbluth, Phys. Rev. 79, 615 (1950).

[2] A.I. Akhiezer and M.P. Rekalo, Sov. J. Part. Nucl. 4, 277 (1974).

[3] M.K. Jones, et al., Phys. Rev. Lett. 84, 1398 (2000). J. Arrington, PRC 68, 034325 (2003), A.J.R. Puckett, et al., Phys. Rev. Lett. 104242301 (2010).

[4] C.E. Carlson and M. Vanderhaeghen Annu. Rev. Nucl. Part. Sci. 5, 171 (2007), J. Arrington, P.G. Blunden and W. Melnitchouk Prog. Part. Nucl. Phys. 66, 782 (2011).

[5] J. Mar, et al., Phys. Rev. Lett. 21482 (1968); J. Arrington, Phys. Rev. C69, 032201 (2004).

[6] J. Arrington et al., arXiv:nucl-ex/0408020; D.M. Nikolenko et al., PoS (ICHEP 2010) 164; A. V. Gramolin et al., Nucl. Phys. B (Proc. Suppl.) 225-227 216 (2012).

[7] http://www.inp.nsk.su/ gramolin/esepp.html

[8] V.S. Fadin, R.E. Gerasimov, and A.L. Feldman, private communication.

[9] J. Arrington and I. Sick, Phys. Rev. C70 028203 (2004).

[10] P.G. Blunden, W. Melnitchouk and J.A. Tjon, Phys. Rev. C72 034612 (2005).

[11] I. A. Qattan, A. Alsaad, and J. Arrington, Phys. Rev. C84 054317 (2011)

[12] E. Tomasi-Gustafsson et al., arXiv:0909.4736

[13] J. Arrington, W. Melnitchouk, and J. Tjon, Phys. Rev. C76 035205 (2007) 